\title{
Variabilidad de la Síntesis de RANKL por Linfocitos $T$ frente a Distintos Serotipos Capsulares de Porphyromonas gingivalis
}

\author{
Variability in the RANKL Synthesis by T Lymphocytes in \\ Response to Different Porphyromonas gingivalis Capsular Serotypes
}

Navarrete $M^{1}$, Silva $A^{2}$, Sanz $M^{3}$, Vernal $R^{1}$

\begin{abstract}
RESUMEN
Propósito: Las periodontitis representan un grupo heterogéneo de infecciones periodontales cuya etiología son las bacterias residentes en el biofilm subgingival. Aunque este biofilm está constituido por una amplia variedad de especies bacterianas, sólo un número limitado de especies, como Porphyromonas gingivalis, se ha asociado a la etiología de la enfermedad. $P$. gingivalis expresa diversos factores de virulencia que pueden causar daño directo a los tejidos del hospedero; sin embargo, su mayor patogenicidad involucra la inducción de una respuesta inmuno-inflamatoria, durante la cual se secretan una amplia variedad de citoquinas, quimioquinas y mediadores inflamatorios que pueden inducir la destrucción de los tejidos de soporte de los dientes y la pérdida de ellos. Método: En esta investigación, se evaluó si los distintos serotipos capsulares (K) de $P$. gingivalis pueden determinar los niveles de síntesis de RANKL, citoquina clave en la destrucción del hueso alveolar durante la periodontitis. Para ello, se cuantificaron los niveles de expresión de RANKL mediante PCR cuantitativa y los niveles de secreción mediante ELISA en linfocitos T activados en presencia de los serotipos capsulares K1-K6 de $P$. gingivalis, y estos se correlacionaron a los niveles de expresión de los factores de transcripción asociados a cada uno de los fenotipos de linfocitos efectores: Th1 (T-bet), Th2 (GATA-3), Th17 (RORC2) y Treg (Foxp3). Resultados: Mayores niveles de expresión y secreción de RANKL fueron detectados en linfocitos T activados en presencia de los serotipos K1 y K2 de $P$. gingivalis, en comparación a los detectados ante los otros serotipos. Además, estos mayores niveles de RANKL se correlacionaron positivamente con los niveles de expresión de RORC2. Conclusión: Estos datos demuestran que la síntesis de RANKL por linfocitos T se restringe a ciertos serotipos capsulares de $P$. gingivalis (K1 y K2) y permiten sugerir que los serotipos $\mathrm{K} 1$ y K2 de $P$. gingivalis podrían asociarse a la destrucción del hueso alveolar y a la pérdida de los dientes durante la periodontitis.
\end{abstract}

Rev. Clin. Periodoncia Implantol. Rehábil. Oral Vol. 3(1); 19-23, 2010.

Palabras clave: RANKL, Porphyromonas gingivalis, Th17, periodontitis, linfocitos T.

\section{ABSTRACT}

Aim: Periodontitis represents a heterogenic group of periodontal infections elicited by bacteria residing at the subgingival biofilm. Although this biofilm is constituted by a broad variety of bacterial species, only a limited number has been associated with the periodontitis aetiology, among them Porphyromonas gingivalis. $P$. gingivalis express a number of virulence factors that contribute to direct tissue damage; however, their pathogenicity relies mainly on the induction of a host immuno-inflammatory response. This leads to the release of a broad array of cytokines, chemokines and inflammatory mediators, which cause destruction of the tooth-supporting alveolar bone and ultimately tooth loss. Method: In the present investigation, in order to determine whether different $P$. gingivalis serotypes might lead to a differential RANKL synthesis, a key cytokine involved in alveolar bone resorption, the mRNA expression and secretion of RANKL and the expression of transcription factors T-bet, GATA-3, RORC2 and Foxp3, the master-switch genes controlling the Th1, Th2, Th17, and Treg cell differentiation, respectively, were analyzed on human T cells activated with different $P$. gingivalis capsular (K) serotypes. Results: T lymphocytes responding to $P$. gingivalis serotypes $\mathrm{K} 1$ or $\mathrm{K} 2$, but not to the other serotypes, led to an increased expression and secretion of RANKL. In addition, these higher RANKL levels correlate with RORC2 expression upon activation with K1 or K2 serotypes. Conclusion: These data demonstrated that RANKL expression and secretion by T lymphocytes was restricted to particular $P$. gingivalis serotypes (namely K1 and K2), and allowed to suggest a link between these serotypes with alveolar bone destruction and teeth loosening during the periodontitis.

Rev. Clin. Periodoncia Implantol. Rehábil. Oral Vol. 3(1); 19-23, 2010.

Key words: RANKL, Porphyromonas gingivalis, Th17, periodontitis, T lymphocytes.

\section{INTRODUCCIÓN}

Porphyromonas gingivalis es un cocobacilo, Gram-negativo, pigmentado de negro, asacarolítico y anaerobio estricto, perteneciente al género Porphyromonas de la familia Bacteroidaceae $e^{(1-4)}$. Numerosas líneas de investigación evidencian clínicamente el rol etiológico de $P$. gingivalis en la periodontitis, principalmente en la forma crónica de la enfermedad( ${ }^{(5-12)}$, y su identificación en el saco periodontal se ha asociado directamente a la destrucción tisular progresiva ${ }^{(13,14)}$. Adicionalmente, $P$. gingivalis se ha identificado como un factor asociado en ciertas condiciones sistémicas, tal como ateroesclerosis, trastornos cardiacos, neumonía, preeclamsia y parto prematuro(15-20), y diversos esfuerzos se han llevado a cabo para desarrollar vacunas dirigidas a lograr inmunización contra esta especie bacteriana ${ }^{(21-23)}$

Basado en los antígenos de la cápsula extracelular, se han descrito 6 serotipos distintos de $P$. gingivalis y se han denominado de $\mathrm{K} 1$ a $\mathrm{K} 6^{(24,25)}$. Al inocular en forma subcutanéa estos 6 serotipos capsulares de $P$. gingivalis en animales de experimentación $(0,1 \mathrm{~mL}$ de una suspensión celular conteniendo $5 \times 10^{10}$ bacterias $/ \mathrm{mL}$ ), se originaron flegmones, la mayoría acompañados de necrosis y/o ulceración de

1. Laboratorio de Biología Periodontal, Departamento de Odontología Conservadora, Facultad de Odontología, Universidad de Chile, Santiago, Chile. 2. Departamento de Medicina Celular y Molecular, Centro de Investigaciones Biológicas, Consejo Superior de Investigaciones Científicas (CSIC), Madrid, España.

3. Departamento de Estomatología III, Facultad de Odontología, Universidad Complutense de Madrid, Madrid, España.

Correspondecia autor: Rolando Vernal. Laboratorio de Biología Periodontal, Facultad de Odontología, Universidad de Chile. Sergio Livingstone 943, Independencia, Santiago, Chile. Fono: 56-2-9781815 / Fax: 56-2-9781833. rvernal@uchile.cl Trabajo recibido el 31/03/2010. Aprobado para su publicación el 18/04/2010. 
la piel, a diferencia de las lesiones menos severas provocadas por la cepa 381 de $P$. gingivalis carente de cápsula, la que provocó abscesos localizados subcutáneos, sugiriendo un rol relevante de la cápsula en la virulencia bacteriana(26)

En pacientes afectados por periodontitis, se ha demostrado diferencias de prevalencia y distribución entre los distintos serotipos de $P$. gingivalis ${ }^{(27,28)}$. Además, se ha observado la capacidad de adhesión a cultivos de células epiteliales obtenidas de sacos periodontales de pacientes con periodontitis, detectándose mayor capacidad adhesiva del serotipo K4(29). Una composición diferente en moléculas de hidratos de carbono se ha establecido entre las cápsulas de los serotipos $\mathrm{K} 1$ y $\mathrm{K}^{(30,31)}$ y se ha evidenciado una mayor capacidad inmuno-estimuladora en macrófagos murinos ${ }^{(32)}$ y mayor resistencia a la fagocitosis y lisis por neutrófilos ${ }^{(33)}$ de la cepa $\mathrm{K} 1$, demostrando un rol modulador de la respuesta inmune innata y sugiriendo un mayor potencial virulento de esta cepa bacteriana en comparación a las otras.

Debido a que ciertos tipos capsulares de $P$. gingivalis son capaces de modular la respuesta inmune innata, es posible especular que el tipo de cápsula presente podría también desempeñar un rol relevante en la inducción de la respuesta inmune adaptativa, la síntesis de citoquinas y la destrucción de los tejidos durante la periodontitis. En el presente trabajo, linfocitos T humanos fueron estimulados con diferentes concentraciones bacterianas de los distintos serotipos capsulares de $P$. gingivalis y se analizaron los niveles de la citoquina ligando del receptor activador del factor nuclear $\mathrm{KB}$ (RANKL), determinante clave de la destrucción del hueso alveolar durante la periodontitis ${ }^{(34-38)}$

\section{MATERIAL Y MÉTODOS}

\section{Cepas de Porphyromonas gingivalis}

Para el presente estudio se utilizaron las especies bacterianas en las que originalmente fueron descritos los distintos serotipos capsulares de $P$. gingivalis ${ }^{(24,25)}$. Los cultivos bacterianos de las cepas W83 (K1), HG184 (K2), A7A1-28 (K3), ATCC 49417 (K4), HG1690 (K5), HG1691 (K6) y de la cepa ATCC 33277 carente de cápsula extracellular (K) fueron realizados como se ha descrito previamente ${ }^{(39)}$.

\section{Curvas de crecimiento bacteriano}

Las curvas de crecimiento bacteriano fueron construídas para obtener un número confiable de unidades formadoras de colonia (UFC) para la estimulación celular ${ }^{(39)}$. Cada cepa bacteriana fue inoculada en $10 \mathrm{~mL}$ de medio de cultivo hasta alcanzar una densidad óptica (DO) de 0,05 medida en un espectrofotómetro a una longitud de onda de $550 \mathrm{~nm}$ (Spectronic 20, Bausch \& Lomb, Rochester, NY, USA). Cada 2 a 5 horas, dependiendo de la especie bacteriana, se realizaron lecturas sucesivas de DO, $100 \mu \mathrm{L}$ de medio de cultivo fueron diluidos seriadamente en PBS (log10) y $100 \mu \mathrm{L}$ de cada dilución fueron sembrados para posterior cuantificación de UFC/mL. El experimento se completó cuando cada especie bacteriana alcanzó la fase estacionaria de crecimiento. Después de 3 a 7 días de incubación, las colonias bacterianas fueron cuantificadas y se confeccionaron las gráficas de las curvas tiempo de cultivo versus DO y UFC/mL versus DO.

\section{Sujetos y muestras}

Mediante punción braquial, $30 \mathrm{~mL}$ de sangre periférica fueron obtenidos de 30 donantes adultos, voluntarios y sanos en el Centro de Transfusiones de la Comunidad de Madrid del Ministerio de Sanidad de España. Las muestras fueron transportadas inmediatamente en condiciones isotérmicas para proceder a la separación celular.

\section{Diferenciación y estimulación de las células dendríticas}

A partir de la muestras de sangre, monocitos fueron purificados y posteriormente diferenciados a células dendríticas (CDs) para ser estimuladas in vitro como se ha descrito previamente ${ }^{(39,40)}$. Utilizando una gradiente de Ficoll (Ficoll-Paque Plus, Amersham Pharmacia Biotech AB, Uppsala, Sweden) y siguiendo protocolos estándar se aislaron las células mononucleares sanguíneas y a partir de ellas se purificaron monocitos mediante separación celular magnética (MACS; Miltenyi Biotec, Bergisch Gladbach, Germany) y utilizando anticuerpos antiCD14 (Miltenyi Biotec). Para la diferenciación de CDs, $10^{6}$ monocitos $/ \mathrm{mL}$ fueron cultivados en medio RPMI-1640, conteniendo suero fetal de cabra 10\% (Gibco Invitrogen Corp., Grand Island, NY, USA) y suplementado con rhGM-CSF y rhlL $1.000 \mathrm{U} / \mathrm{ml}$ (Immunotools, Friesoythe, Germany), e incubados durante 6 días a $37^{\circ} \mathrm{C}$ en un ambiente de $\mathrm{CO}_{2} 5 \%$. Una vez diferenciadas, $10^{6} \mathrm{CDs} / \mathrm{mL}$ fueron estimuladas a concentraciones bacterianas ascendentes (MOls, del inglés: multiplicity of infections) de 0,1 a $10^{3}$ (bacterias/CDs) con los serotipos $\mathrm{K} 1$ a $\mathrm{K} 6$ de $P$. gingivalis durante 2 días. CDs estimuladas con la cepa $\mathrm{K}^{-}$de $P$. gingivalis o $10 \mathrm{ng} /$ $\mathrm{mL}$ de LPS de Escherichia coli 0111:B4 (Fluka, Sigma-Aldrich Chemie, Buchs, Switzerland) fueron usadas para la comparación y CDs sin estimular fueron utilizadas como control.

\section{Purificación y activación de los linfocitos T}

Linfocitos $\mathrm{T} \mathrm{CD4}^{+}$se obtuvieron a partir de las células mononucleares sanguíneas mediante depleción inmunomagnética siguiendo las indicaciones del fabricante (MACS; Miltenyi Biotec) y fueron congeladas a $-160^{\circ} \mathrm{C}$ para su posterior uso. Para la activación celular $10^{6}$ linfocitos $\mathrm{T} / \mathrm{mL}$ fueron cultivados junto a CDs autólogas estimuladas (50:1) en medio RPMI-1640 conteniendo suero fetal de cabra $10 \%$ por 5 días a $37^{\circ} \mathrm{C}$. Linfocitos $\mathrm{T}$ cultivados libres de CDs o cultivados junto a CDs sin estimular fueron utilizados como control.

\section{Análisis del fenotipo celular}

La purificación de los monocitos, la diferenciación y estimulación de las CDs y la purificación y activación de los linfocitos T $\mathrm{CD}^{+}$fueron evaluadas mediante citometría de flujo como se ha descrito previamente ${ }^{(35)}$. Las células fueron incubadas durante $20 \mathrm{~min}$ a $4^{\circ} \mathrm{C}$ y en la oscuridad con anticuerpos monoclonales anti-CD1a, CD4, CD14, CD25, CD44, CD80, CD83, CD86, CD62L, CD69 y HLA-DR conjugados con PE, FITC o PE/Cy5 (BD Biosciences Pharmingen, San José, CA, USA) y posteriormente analizadas mediante citometría de flujo (EPICS $\mathrm{XL}$, Beckman Coulter, Fullerton, CA, USA).

\section{PCR cuantitativa}

El ARNm citoplasmático total fue aislado de los linfocitos T CD4 ${ }^{+}$ activados utilizando el método NP-40 como se ha descrito previamente ${ }^{(41)}$ La trascripción reversa fue realizada usando el kit Transcriptor First Strand cDNA synthesis, de acuerdo a las instrucciones del fabricante (Roche Applied Science, Mannheim, Germany). Para cuantificar los niveles de RANKL y de los factores de transcripción asociados a cada uno de los fenotipos linfocitarios efectores (Th1: T-bet, Th2: GATA-3, Th17: RORC2 y Treg: Foxp3), $50 \mathrm{ng}$ de ADNc fueron amplificados utilizando partidores y sondas específicos (Tabla 1), un kit FastStart Taqman Probe Master (Roche) y un termociclador en tiempo real ABI PRISM 7900 Sequence Detector System (Applied Biosystems, Foster City, CA, USA) como se ha descrito previamente ${ }^{(41)}$. En todos los casos, los niveles de la subunidad ARNr $18 \mathrm{~S}$ fueron usados como control endógeno de expresión.

Tabla 1. Partidores y sondas utilizadas en los experimentos de PCR cuantitativa.

\begin{tabular}{|l|c|c|c|}
\hline \hline ARNm & Forward Primer & Reverse Primer & Sonda \\
\hline RANKL & tgattcatgtaggagaattaaacagg & gatgtgctgtgatccaacga & 17 \\
\hline T-bet & tccaagtttaatcagcaccaga & tgacaggaatgggaacatcc & 9 \\
\hline GATA-3 & ctcattaagcccaagcgaag & tctgacagttcgcacaggac & 71 \\
\hline RORC2 & agaaggacagggagccaag & caagggatcacttcaatttgtg & 21 \\
\hline Foxp3 & Acctacgccacgctcatc & tcattgagtgtccgctgct & 50 \\
\hline ARNr 18S & ctcaacacgggaaacctcac & cgctccaccaactaagaacg & 77 \\
\hline
\end{tabular}

\section{ELISA}

Después de 5 días de estimulación, el sobrenadante de los cultivos de linfocitos $\mathrm{T} C D 4^{+}$fue colectado y la secreción de RANKL fue cuantificada mediante ELISA de acuerdo al protocolo del fabricante (Quantikine $®, R \& D$ Systems Inc., Minneapolis, MN, USA) y utilizando un espectrofotómetro a 492 nm (Labsystem Multiskan, Helsinki, Finland).

\section{Análisis de los datos}

Los datos de citometría de flujo se analizaron utilizando el programa computacional WinMDi 2.9 (The Scripps Research Institute, La Jolla, CA, USA), se representaron como histogramas y se expresaron como porcentaje de células positivas. Los datos de PCR cuantitativa se analizaron con el programa ABI PRISM (Applied Biosystems). La cuantificación de expresión relativa se obtuvo utilizando el método $2^{-\Delta \Lambda C t}$, normalizando la expresión de los ARNm a la expresión del ARNr 18S y comparando con la expresión normalizada en CDs no estimuladas ${ }^{(38)}$. Los datos se expresaron como media \pm desviación estándar y se analizaron estadísticamente utilizando el paquete estadístico SPSS 15.0 (Lead Technologies Inc., Charlote, NC, USA). La normalidad de la distribución de los datos se determinó utilizando la prueba de Shapiro- 
Wilk. Los datos de citometría de flujo se analizaron con la prueba de Chicuadrado, los datos de PCR cuantitativa con las pruebas ANOVA y posthoc de Tukey y los datos de ELISA se analizaron utilizando la prueba $\mathrm{U}$ de Mann-Whitney. Los coeficientes de correlación se obtuvieron utilizando la prueba $r$ de Pearson. Se consideraron las diferencias como estadísticamente significativas cuando $p$-value $<0,05$.

\section{RESULTADOS}

En linfocitos $T$ activados con CDs autólogas previamente estimuladas, a distintas concentraciones bacterianas de los distintos serotipos capsulares de $P$. gingivalis, se determinó la expresión de RANKL evaluando los niveles del ARNm mediante PCR cuantitativa en tiempo real (Figura 1). Los datos fueron representados como expresión relativa y revelaron un incremento dosis-dependiente en la expresión de RANKL con respecto a la carga bacteriana de estimulación. Los serotipos $\mathrm{K} 1$ y K2 de $P$. gingivalis indujeron significativamente mayores niveles de expresión de RANKL a todas las concentraciones de carga bacteriana, en comparación a los otros serotipos capsulares y a la cepa K- carente de cápsula extracelular.

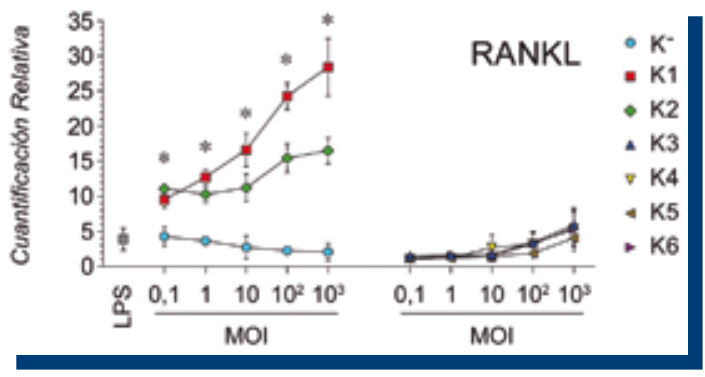

Figura 1. Niveles de expresión de RANKL. Análisis mediante PCR cuantitativa de la expresión de RANKL en linfocitos T activados con células dendríticas estimuladas con distintas concentraciones de carga bacteriana de los serotipos capsulares K1 a K6 y de la cepa no encapsulada K de Porphyromonas gingivalis. El cuadro gris corresponde a los niveles de expresión de RANKL en linfocitos T activados en presencia de LPS de Escherichia coli, usados como control positivo (LPS). Los datos se representan como promedio \pm desviación estándar de 10 experimentos independientes. * $p<0,05$.

La sobre expresión de RANKL fue confirmada a nivel proteico cuando se evaluó su secreción mediante ELISA (Figura 2). Se detectaron mayores niveles de secreción de RANKL cuando los linfocitos T fueron activados en presencia de los serotipos $\mathrm{K} 1$ y K2, en comparación a los otros serotipos capsulares y a la cepa $\mathrm{K}^{-}$de $P$. gingivalis.

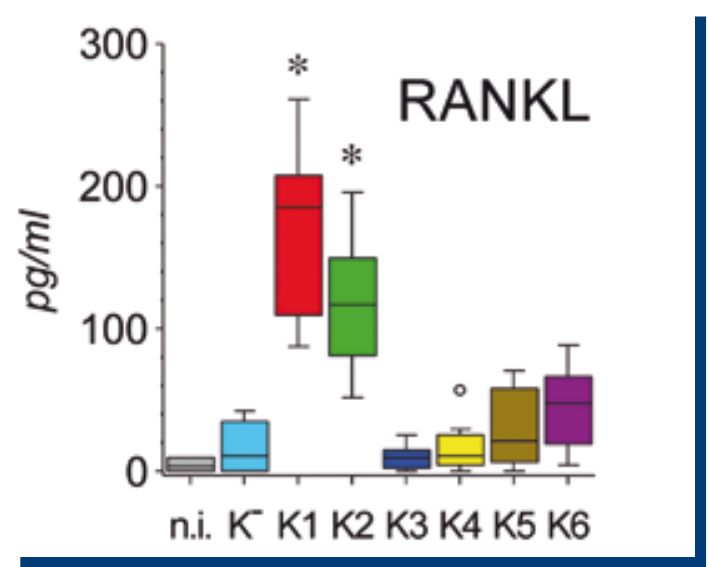

Figura 2. Niveles de secreción de RANKL. Los niveles secretados de RANKL se cuantificaron mediante ELISA en sobrenadantes de cultivo de linfocitos $T$ activados con células dendríticas estimuladas con $10^{9} \mathrm{UFC} / \mathrm{mL}\left(\mathrm{MOI}=10^{3}\right)$ de los serotipos capsulares K1 a K6 y de la cepa no encapsulada K- de Porphyromonas gingivalis. Los niveles de RANKL en linfocitos T expuestos a células dendríticas no inducidas fueron usados como control (n.i.). Los datos son expresados como concentración de RANKL (pg/mL) para 10 experimentos independientes. Las cajas muestran la mediana y el recorrido inter-cuartílico, las pestañas los percentiles 10 y 90 y el círculo (K4) un dato extremo. ${ }^{*} \mathrm{p}<0,05$.
El análisis de correlación entre los niveles de expresión de los factores de transcripción asociados a los fenotipos efectores Th1, Th2, Th17 y Treg y los niveles de secreción de RANKL reveló una correlación positiva con el factor de transcripción RORC2 (Th17) cuando los linfocitos T fueron activados en presencia de los serotipos $\mathrm{K} 1$ y K2 de $P$. gingivalis (para K1 $p=0,029$ y para $K 2 p=0,102$ ). Cuando los linfocitos $T$ fueron estimulados en presencia de los serotipos K3-K6 y la cepa K- de $P$. gingivalis, no se observó correlación entre los niveles secretados de RANKL y los expresados de los distintos factores de transcipción (datos no mostrados).

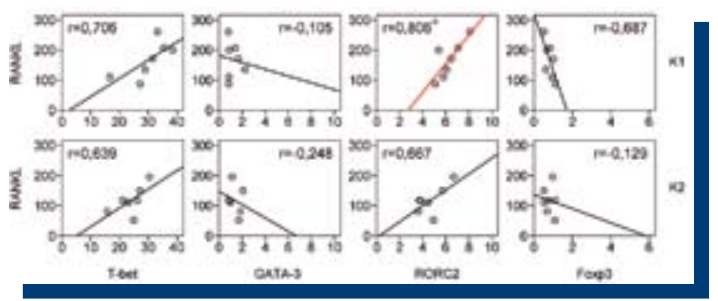

Figura 3. Correlación entre RANKL y T-bet, GATA-3, RORC2 y Foxp3. Análisis de correlación de los niveles secretados de RANKL y los expresados de T-bet, GATA-3, RORC2 y Foxp3 en linfocitos T activados con células dendríticas estimuladas con $10^{\circ}$ UFC/mL $\left(\mathrm{MOI}=10^{3}\right)$ de los serotipos capsulares K1 y K2 de Porphyromonas gingivalis. $p<0,05$.

\section{DISCUSIÓN}

La respuesta inmune desarrollada frente al biofilm subgingival es compleja y la definición del fenotipo linfocitario efector asociado a la patogenia de la destrucción tisular durante la periodontitis es aún objeto de una amplia investigación. En efecto, diversos estudios han asociado tanto una respuesta linfocitaria efectora tipo Th1 como una tipo Th2 a la progresión de la enfermedad periodontal ${ }^{(42-55)}$. Además, durante los últimos años, tanto el fenotipo Th17 como el Treg han sido implicados en la patogenia de la periodontitis ${ }^{(56-63)}$

Trabajos previos de nuestro grupo de investigación han evidenciado una respuesta asociada tanto a un fenotipo Th1 como a un fenotipo Th17 durante la progresión de la periodontitis ${ }^{(35,44,57,64)}$. En las lesiones periodontales activas se detectó un incremento de la síntesis de las citoquinas asociadas a la destrucción ósea alveolar (IL-1 $\beta$, IL-17, IFN$\gamma$, TNF- $\alpha$ y RANKL), de la síntesis de la metaloproteinasa-13, asociada a la destrucción de las fibras de colágeno del ligamento periodontal, y de la expresión de los factores de transcripción T-bet, RORC2 y Foxp3, en comparación con lesiones inactivas caracterizadas por la ausencia de actividad de la enfermedad periodontal(35,44,57,64-66). Además, estos mayores niveles se asociaron a la presencia de $P$. gingivalis y $A$. actinomycetemcomitans en el biofilm subgingival local(64).

Se ha establecido que el perfil de citoquinas sintetizado en respuesta a las bacterias periodontales depende, al menos en parte, del número y del tipo de microorganismos que la desencadena ${ }^{(67-70)}$. En este contexto, se ha propuesto que las bacterias podrían definir la polarización de la respuesta efectora Th y consecuentemente la secreción de un patrón de citoquinas bacteria-específico ${ }^{(71,72)}$. Esta especialización efectora podría implicar una polarización global de la respuesta inmune, iniciándose con las células que tienen el primer contacto con los antígenos ${ }^{(73)}$.

La cápsula bacteriana desempeña un papel importante en la virulencia de las bacterias Gram-positivas y Gram-negativas. Las cepas de $P$. gingivalis que presentan cápsula producen patologías más severas y extensas en animales de experimentación ${ }^{(26,74-76)}$, poseen una mayor actividad proteolítica ${ }^{(77-79)}$, inducen una respuesta inflamatoria más intensa ${ }^{(28,76)}$ y mayor destrucción ósea alveolar ${ }^{(80)}$ en comparación con las cepas carentes de cápsula.

Los serotipos $\mathrm{K} 1$ y $\mathrm{K} 2$ de $P$. gingivalis poseen un mayor potencial inmuno-estimulador de CDs comparado con los otros serotipos capsulares ${ }^{(39)}$ y este mayor potencial es similar al previamente descrito para $A$. actinomycetemcomitans ${ }^{(40)}$. Además, recientemente se han demostrado diferencias de inmunogenicidad entre los distintos serotipos capsulares de $P$. gingivalis al inducir en linfocitos $T$ una respuesta efectora distinta (Vernal $\mathrm{R}$, datos sin publicar).

Dado que la progresión de la periodontitis se caracteriza por la destrucción del hueso alveolar ${ }^{(81,82)}$, en linfocitos $\mathrm{T}$ activados en presencia de los distintos serotipos capsulares de $P$. gingivalis analizamos los niveles de expresión y secreción de RANKL, citoquina asociada a este proceso (34-38) Se detectaron niveles significativamente mayores de expresión y secreción de RANKL cuando los linfocitos T fueron estimulados con los serotipos $\mathrm{K} 1$ y K2, y estos mayores niveles correlacionaron positivamente con los del factor de transcripción RORC2 propio del fenotipo celular tipo Th17, sugiriendo una asociación entre los serotipos K1 y K2 de $P$. gingivalis y la destrucción ósea alveolar y la pérdida dentaria, signos característicos de la periodontitis.

Estos datos son consistentes con resultados de otros grupos de investigación que han descrito diferencias entre los distintos serotipos 
capsulares de $P$. gingivalis. Se ha establecido que los serotipos $\mathrm{K} 5$ y K6 se detectan con más frecuencia en los pacientes afectados de periodontitis $^{(27)}$, los serotipos K1 y K3 poseen cápsulas con diferente composición molecular ${ }^{(30,31)}$, el serotipo K4 posee una mayor capacidad adhesiva a células epiteliales obtenidas de sacos periodontales ${ }^{(29)}$, el serotipo K1 induce mayor síntesis de quimioquinas en macrófagos murinos $^{(32)}$ y los serotipos $\mathrm{K} 1$ y K2 muestran mayor resistencia a la fagocitosis y lisis por neutrófilos ${ }^{(33)}$

Variaciones en la estructura de la cápsula extracelular podrían estar involucradas en estas diferencias de inmunogenicidad. Un análisis de polimorfismo del locus PG0106-PG0120 asociado a la síntesis de la cápsula ha evidenciado variaciones significativas entre los distintos serotipos capsulares de $P$. gingivalis ${ }^{(83-85)}$. Además, la ausencia de una pauta abierta de lectura en la cepa $\mathrm{K}^{-}$se ha asociado a la ausencia de cápsula( ${ }^{(86)}$ y su deleción en el serotipo $\mathrm{K} 1$ genera mutantes de $P$. gingivalis que no sintetizan cápsula extracelular ni son reactivos frente a anticuerpos anti-K1 ${ }^{\left({ }^{(83)}\right.}$. Por lo tanto, variaciones genéticas en estos loci podrían reflejarse en diferencias en la composición molecular y estructural de la cápsula ${ }^{(30)}$, y asociarse a las diferencias inmuno-estimuladoras en macrófagos, de adhesión a queratinocitos, de resistencia a la fagocitosis mediada por neutrófilos ${ }^{(29,32,33)}$, y a diferencias en la inducción de RANKL por distintos serotipos capsulares de $P$. gingivalis descritas en el presente estudio.

En $P$. gingivalis, la cápsula es relevante en las interacciones hospedero-patógeno $(28,29,32,76,80)$ y se ha demostrado que algunas formas de la cápsula extracelular le confieren resistencia frente a la fagocitosis por los macrófagos ${ }^{(33)}$. Nuestros datos permiten establecer que el tipo de cápsula presente determina la activación de las CDs y la respuesta efectora de los linfocitos T. Así, puede sugerirse que alterando la proporción de los diferentes serotipos capsulares de $P$. gingivalis en las lesiones periodontales esta especie bacteriana es capaz de manipular tanto la respuesta inmune como la destrucción ósea alveolar a nivel local, modulando una respuesta efectora o una respuesta protectora tisular y de esta forma determinando las características clínicas de la periodontitis.

Hasta el momento no existe evidencia científica que permita establecer una asociación directa entre un tipo clonal particular de $P$ gingivalis y la presencia, tipo o severidad de enfermedad periodontal. Sin embargo, considerando nuestros datos, podemos establecer que las pruebas microbiológicas actuales que analizan la presencia de $P$. gingivalis sólo proporcionan una información parcial de la realidad microbiológica local debido a la variable inmunogenicidad entre sus subtipos séricos capsulares. Las pruebas microbiológicas deben considerar que la virulencia de un patógeno periodontal puede variar entre las distintas cepas y/o distintos serotipos y estas variaciones se asocian tanto a una diferente capacidad de evadir la respuesta inmune del hospedero como a la activación de una respuesta efectora particular.

\section{AGRADECIMIENTOS}

Los autores agradecen al Dr. Arie Jan van Winkelhoff (Universidad y Centro Médico Groningen, Universidad Estatal de Groningen, Holanda) por proporcionarnos los diferentes serotipos capsulares de $P$. gingivalis, al Dr. Rubén León (Facultad de Odontología, Universidad de Chile, Chile) y a la Dra. María del Carmen Sánchez (Facultad de Odontología, Universidad Complutense de Madrid, España) por la colaboración en el trabajo con cultivos bacterianos y al Dr. Pedro Lastres (Centro de Investigaciones Biológicas, Consejo Superior de Investigaciones Científicas, España) por su ayuda con los análisis mediante citometría de flujo. Este trabajo fue financiado por el proyecto FIS-060181 del Ministerio de Salud de España y formó parte de la tesis de investigación conducente al grado de Doctor en Ciencias Odontológicas de la Universidad Complutense de Madrid de RV, quien fue financiado por beca CONICYT-26080046 del Gobierno de Chile.

\section{REFERENCIAS BIBLIOGRÁFICAS}

1. Hajishengallis G. Porphyromonas gingivalis-host interactions: open war or intelligent guerilla tactics? Microbes and Infection. 2009; 11: 637-45. 2. Holt SC y Ebersole JL. Porphyromonas gingivalis, Treponema denticola, and Tannerella forsythia: the "red complex", a prototype polybacterial pathogenic consortium in periodontitis. Periodontology 2000. 2005; 38: 72-122

3. White $D$ y Mayrand D. Association of oral Bacteroides with gingivitis and adult periodontitis. Journal of Periodontal Research. 1981; 16: 259-65.

4. Sanz M, Lau L, Herrera D y cols. Methods of detection of Actinobacillus actinomycetemcomitans, Porphyromonas gingivalis and Tannerella forsythensis in periodontal microbiology, with special emphasis on advanced molecular techniques: a review. Journal of Clinical Periodontology. 2004; 31: 1034-47.

5. Zambon JJ. Periodontal diseases: microbial factors. Annals of Periodontology. 1996; 1: 879-925.

6. van Winkelhoff AJ, van Steenbergen TJ y de Graaff J. The role of black-pigmented Bacteroides in human oral infections. Journal of Clinical Periodontology. 1988; 15: 145-55

7. van WinkelhoffAJ, Loos BG, van der Reijden WA y cols. Porphyromonas gingivalis, Bacteroides forsythus and other putative periodontal pathogens in subjects with and without periodontal destruction. Journal of Clinical Periodontology. 2002; 29: 1023-8.

8. Slots $\mathrm{J} y$ Ting $\mathrm{M}$. Actinobacillus actinomycetemcomitans and Porphyromonas gingivalis in human periodontal disease: occurrence and treatment. Periodontology 2000. 1999; 20: 82-121.

9. Socransky SS y Haffajee AD. Evidence of bacterial etiology: a historical perspective. Periodontology 2000. 1994; 5: 7-25

10. Slots J. Bacterial specificity in adult periodontitis. A summary of recent work. Journal of Clinical Periodontology. 1986; 13: 912-7.

11. Holt SC, Kesavalu L, Walker $S$ y cols. Virulence factors of Porphyromonas gingivalis. Periodontology 2000. 1999; 20: 168-238.

12. SocranskySS, HaffajeeAD, Ximenez-Fyvie LAy cols. Ecological considerations in the treatment of Actinobacillus actinomycetemcomitans and Porphyromonas gingivalis periodontal infections. Periodontology 2000. 1999; 20: 341-62

13. Dzink JL, Socransky SS y Haffajee AD. The predominant cultivable microbiota of active and inactive lesions of destructive periodontal diseases. Journal of Clinical Periodontology. 1988; 15: 316-23.

14. Dzink JL, Tanner AC, Haffajee AD y cols. Gram negative species associated with active destructive periodontal lesions. Journal of Clinica Periodontology. 1985; 12: 648-59

15. Seymour GJ, Ford PJ, Cullinan MP y cols. Relationship between periodontal infections and systemic disease. Clinical Microbiology and Infection. 2007; 13 Suppl 4: 3-10.

16. Gibson FC, Yumoto $\mathrm{H}$, Takahashi $\mathrm{Y}$ y cols. Innate immune signaling and Porphyromonas gingivalis-accelerated atherosclerosis. Journal of Dental Research. 2006; 85: 106-21.

17. Okuda K, Kimizuka R, Abe S y cols. Involvement of periodontopathic anaerobes in aspiration pneumonia. Journal of Periodontology. 2005; 76: 2154-60.
18. Yamazaki K, Honda T, Domon H y cols. Relationship of periodontal infection to serum antibody levels to periodontopathic bacteria and inflammatory markers in periodontitis patients with coronary heart disease. Clinical and Experimental Immunology. 2007; 149: 445-52.

19. Leon R, Silva N, Ovalle Ay cols. Detection of Porphyromonas gingivalis in the amniotic fluid in pregnant women with a diagnosis of threatened premature labor. Journal of Periodontology. 2007; 78: 1249-55

20. Contreras A, Herrera JA, Soto JE y cols. Periodontitis is associated with preeclampsia in pregnant women. Journal of Periodontology. 2006; 77: 182-8.

21. Gibson FC y Genco CA. Prevention of Porphyromonas gingivalisinduced oral bone loss following immunization with gingipain R1. Infection and Immunity. 2001; 69: 7959-63.

22. Rajapakse PS, O'Brien-Simpson NM, Slakeski N y cols. Immunization with the RgpA-Kgp proteinase-adhesin complexes of Porphyromonas gingivalis protects against periodontal bone loss in the rat periodontitis model. Infection and Immunity. 2002; 70: 2480-6.

23. Nakagawa T, Sims T, Fan $Q$ y cols. Functional characteristics of antibodies induced by Arg-gingipain (HRgpA) and Lys-gingipain (Kgp) from Porphyromonas gingivalis. Oral Microbiology and Immunology. 2001; 16: 202-11

24. van Winkelhoff AJ, Appelmelk BJ, Kippuw $\mathrm{N}$ y cols. K-antigens in Porphyromonas gingivalis are associated with virulence. Oral Microbiology and Immunology. 1993; 8: 259-65.

25. Laine ML, Appelmelk BJ y van Winkelhoff AJ. Novel polysaccharide capsular serotypes in Porphyromonas gingivalis. Journal of Periodonta Research. 1996; 31: 278-84.

26. Laine ML y van Winkelhoff AJ. Virulence of six capsular serotypes of Porphyromonas gingivalis in a mouse model. Oral Microbiology and Immunology. 1998; 13: 322-5.

27. Laine ML, Appelmelk BJ y van Winkelhoff AJ. Prevalence and distribution of six capsular serotypes of Porphyromonas gingivalis in periodontitis patients. Journal of Dental Research. 1997; 76: 1840-4.

28. Califano JV, Schifferle RE, Gunsolley JC y cols. Antibody reactive with Porphyromonas gingivalis serotypes K1-6 in adult and generalized earlyonset periodontitis. Journal of Periodontology. 1999; 70: 730-5.

29. Dierickx K, Pauwels M, Laine ML y cols. Adhesion of Porphyromonas gingivalis serotypes to pocket epithelium. Journal of Periodontology. 2003; 74: 844-8.

30. Farquharson SI, Germaine GR y Gray GR. Isolation and characterization of the cell-surface polysaccharides of Porphyromonas gingivalis ATCC 53978. Oral Microbiology and Immunology. 2000; 15: 151-7.

31. Schifferle RE, Reddy MS, Zambon JJ y cols. Characterization of a polysaccharide antigen from Bacteroides gingivalis. J Immunol. 1989 143: 3035-42.

32. d'Empaire G, Baer MT y Gibson FC. The K1 serotype capsular polysaccharide of Porphyromonas gingivalis elicits chemokine production from murine macrophages that facilitates cell migration. Infection and Immunity. 2006; 74: 6236-43.

33. Sundqvist $G$, Figdor D, Hanstrom $L$ y cols. Phagocytosis and virulence 
of different strains of Porphyromonas gingivalis. Scandinavian Journal of Dental Research. 1991; 99: 117-29.

34. Vernal R, Chaparro A, Graumann R y cols. Levels of cytokine receptor activator of nuclear factor kappaB ligand in gingival crevicular fluid in untreated chronic periodontitis patients. Journal of Periodontology. 2004; 75: 1586-91

35. Vernal R, Dutzan N, Hernandez M y cols. High expression levels of receptor activator of nuclear factor-kappa $B$ ligand associated with human chronic periodontitis are mainly secreted by CD4+ T lymphocytes. Journal of Periodontology. 2006; 77: 1772-80.

36. Crotti T, Smith MD, Hirsch R y cols. Receptor activator NF kappaB ligand (RANKL) and osteoprotegerin (OPG) protein expression in periodontitis. Journal of Periodontal Research. 2003; 38: 380-7.

37. Liu D, Xu JK, Figliomeni L y cols. Expression of RANKL and OPG mRNA in periodontal disease: possible involvement in bone destruction. International Journal of Molecular Medicine. 2003; 11: 17-21.

38. Vernal R, Dezerega A, Dutzan N y cols. RANKL in human periapical granuloma: possible involvement in periapical bone destruction. Oral Diseases. 2006; 12: 283-9.

39. Vernal R, Leon R, Silva A y cols. Differential cytokine expression by human dendritic cells in response to different Porphyromonas gingivalis capsular serotypes. Journal of Clinical Periodontology. 2009; 36: 823-9. 40. Vernal R, Leon R, Herrera D y cols. Variability in the response of human dendritic cells stimulated with Porphyromonas gingivalis or Aggregatibacter actinomycetemcomitans. Journal of Periodontal Research. 2008; 43: 689-97.

41. Vernal R, Velasquez E, Gamonal J y cols. Expression of proinflammatory cytokines in osteoarthritis of the temporomandibular joint. Archives of Oral Biology. 2008; 53: 910-5.

42. Salvi GE, Brown CE, Fujihashi K y cols. Inflammatory mediators of the terminal dentition in adult and early onset periodontitis. Journal of Periodontal Research. 1998; 33: 212-25.

43. Baker PJ, Dixon M, Evans RT y cols. CD4(+) T cells and the proinflammatory cytokines gamma interferon and interleukin- 6 contribute to alveolar bone loss in mice. Infection and Immunity. 1999; 67: 2804-9. 44. Dutzan N, Vernal R, Hernandez M y cols. Levels of interferon-gamma and transcription factor T-bet in progressive periodontal lesions in patients with chronic periodontitis. Journal of Periodontology. 2009; 80: 290-6.

45. Ebersole JL y Taubman MA. The protective nature of host responses in periodontal diseases. Periodontology 2000. 1994; 5: 112-41.

46. Houri-Haddad Y, Wilensky A y Shapira L. T-cell phenotype as a risk factor for periodontal disease. Periodontology 2000. 2007; 45: 67-75.

47. Pradeep AR, Roopa Y y Swati PP. Interleukin-4, a T-helper 2 cell cytokine, is associated with the remission of periodontal disease. Journal of Periodontal Research. 2008; 43: 712-6.

48. Takeichi O, Haber J, Kawai T y cols. Cytokine profiles of T-lymphocytes from gingival tissues with pathological pocketing. Journal of Dental Research. 2000; 79: 1548-55.

49. Gemmell E y Seymour GJ. Cytokines and T cell switching. Critical Reviews in Oral Biology \& Medicine. 1994; 5: 249-79.

50. Bartova J, Kratka-Opatrna Z, Prochazkova J y cols. Th1 and Th2 cytokine profile in patients with early onset periodontitis and their healthy siblings. Mediators of Inflammation. 2000; 9: 115-20.

51. Manhart SS, Reinhardt RA, Payne JB y cols. Gingival cell IL-2 and IL-4 in early-onset periodontitis. Journal of Periodontology. 1994; 65: 807-13.

52. Sigusch B, Klinger G, Glockmann E y cols. Early-onset and adult periodontitis associated with abnormal cytokine production by activated T lymphocytes. Journal of Periodontology. 1998; 69: 1098-104.

53. Fokkema SJ, Loos BG, Slegte C y cols. A type 2 response in lipopolysaccharide (LPS)-stimulated whole blood cell cultures from periodontitis patients. Clinical and Experimental Immunology. 2002; 127: 374-8.

54. Yun PL, Decarlo AA, Collyer C y cols. Hydrolysis of interleukin-12 by Porphyromonas gingivalis major cysteine proteinases may affect local gamma interferon accumulation and the Th1 or Th2 T-cell phenotype in periodontitis. Infection and Immunity. 2001; 69: 5650-60.

55. Reinhardt RA, McDonald TL, Bolton RW y cols. IgG subclasses in gingival crevicular fluid from active versus stable periodontal sites. Journal of Periodontology. 1989; 60: 44-50.

56. Gaffen SL y Hajishengallis G. A new inflammatory cytokine on the block: re-thinking periodontal disease and the Th1/Th2 paradigm in the context of Th17 cells and IL-17. Journal of Dental Research. 2008; 87: 817-28.

57. Vernal R, Dutzan N, Chaparro A y cols. Levels of interleukin-17 in gingival crevicular fluid and in supernatants of cellular cultures of gingival tissue from patients with chronic periodontitis. Journal of Clinical Periodontology. 2005; 32: 383-9.

58. Ohyama H, Kato-Kogoe N, Kuhara A y cols. The involvement of IL23 and the Th17 pathway in periodontitis. Journal of Dental Research. 2009; 88: 633-8.

59. Cardoso CR, Garlet GP, Crippa GE y cols. Evidence of the presence of T helper type 17 cells in chronic lesions of human periodontal disease. Oral Microbiology and Immunology. 2009; 24: 1-6.

60. Takahashi K, Azuma T, Motohira H y cols. The potential role of interleukin-17 in the immunopathology of periodontal disease. Journal of
Clinical Periodontology. 2005; 32: 369-74.

61. Nakajima T, Ueki-Maruyama K, Oda T y cols. Regulatory T-cells infiltrate periodontal disease tissues. Journal of Dental Research. 2005; 84: 639-43.

62. Cardoso CR, Garlet GP, Moreira AP y cols. Characterization of CD4+CD25+ natural regulatory $T$ cells in the inflammatory infiltrate of human chronic periodontitis. Journal of Leukocyte Biology. 2008; 84 311-8.

63. Okui T, Ito H, Honda T y cols. Characterization of CD4+ FOXP3+ T-cell clones established from chronic inflammatory lesions. Oral Microbiology and Immunology. 2008; 23: 49-54.

64. Silva N, Dutzan N, Hernandez M y cols. Characterization of progressive periodontal lesions in chronic periodontitis patients: levels of chemokines, cytokines, matrix metalloproteinase-13, periodontal pathogens and inflammatory cells. Journal of Clinical Periodontology. 2008; 35: 206-14

65. Hernandez M, Valenzuela MA, Lopez-Otin C y cols. Matrix metalloproteinase-13 is highly expressed in destructive periodontal disease activity. Journal of Periodontology. 2006; 77: 1863-70

66. Dutzan N, Gamonal J, Silva A y cols. Over-expression of forkhead box P3 and its association with receptor activator of nuclear factor-kappa B ligand, interleukin (IL) -17, IL-10 and transforming growth factorbeta during the progression of chronic periodontitis. Journal of Clinical Periodontology. 2009; 36: 396-403.

67. Kopitar AN, Ihan Hren $\mathrm{N}$ y Ihan A. Commensal oral bacteria antigens prime human dendritic cells to induce Th1, Th2 or Treg differentiation. Oral Microbiology and Immunology. 2006; 21: 1-5.

68. Kikuchi T, Hahn CL, Tanaka S y cols. Dendritic cells stimulated with Actinobacillus actinomycetemcomitans elicit rapid gamma interferon responses by natural killer cells. Infection and Immunity. 2004; 72: 508996.

69. Kikuchi T, Willis DL, Liu M y cols. Dendritic-NK cell interactions in P. gingivalis-specific responses. Journal of Dental Research. 2005; 84: 858-62.

70. Choi J, Borrello MA, Smith E y cols. Prior exposure of mice to Fusobacterium nucleatum modulates host response to Porphyromonas gingivalis. Oral Microbiology and Immunology. 2001; 16: 338-44.

71. Iwasaki A y Medzhitov R. Toll-like receptor control of the adaptive immune responses. Nature Immunology. 2004; 5: 987-95.

72. Woehrle T, Du W, Goetz A y cols. Pathogen specific cytokine release reveals an effect of TLR2 Arg753GIn during Candida sepsis in humans. Cytokine. 2008; 41: 322-9.

73. Moser $\mathrm{M}$ y Murphy KM. Dendritic cell regulation of TH1-TH2 development. Nature Immunology. 2000; 1: 199-205.

74. van Steenbergen TJ, Kastelein P, Touw JJ y cols. Virulence of blackpigmented Bacteroides strains from periodontal pockets and other sites in experimentally induced skin lesions in mice. Journal of Periodontal Research. 1982; 17: 41-9.

75 Chen PB, Neiders ME, Millar SJ y cols. Effect of immunization on experimental Bacteroides gingivalis infection in a murine model. Infection and Immunity. 1987; 55: 2534-7.

76. Nakano K, Kuboniwa M, Nakagawa I y cols. Comparison of inflammatory changes caused by Porphyromonas gingivalis with distinct fimA genotypes in a mouse abscess model. Oral Microbiology and Immunology. 2004; 19: 205-9.

77. Birkedal-Hansen $\mathrm{H}$, Taylor RE, Zambon JJ y cols. Characterization of collagenolytic activity from strains of Bacteroides gingivalis. Journal of Periodontal Research. 1988; 23: 258-64

78. Neiders ME, Chen PB, Suido $\mathrm{H}$ y cols. Heterogeneity of virulence among strains of Bacteroides gingivalis. Journal of Periodontal Research. 1989; 24: 192-8.

79. Evans RT, Klausen B, Ramamurthy NS y cols. Periodontopathic potential of two strains of Porphyromonas gingivalis in gnotobiotic rats. Archives of Oral Biology. 1992; 37: 813-9.

80. Wilensky A, Polak D, Awawdi S y cols. Strain-dependent activation of the mouse immune response is correlated with Porphyromonas gingivalisinduced experimental periodontitis. Journal of Clinical Periodontology. 2009. 81. Offenbacher S. Periodontal diseases: pathogenesis. Annals of Periodontology. 1996; 1: 821-78.

82. Listgarten MA. Pathogenesis of periodontitis. Journal of Clinical Periodontology. 1986; 13: 418-30.

83. Aduse-Opoku J, Slaney JM, Hashim A y cols. Identification and characterization of the capsular polysaccharide (K-antigen) locus of Porphyromonas gingivalis. Infection and Immunity. 2006; 74: 449-60.

84. Brunner J, Crielaard W y van Winkelhoff AJ. Analysis of the capsular polysaccharide biosynthesis locus of Porphyromonas gingivalis and development of a K1-specific polymerase chain reaction-based serotyping assay. Journal of Periodontal Research. 2008; 43: 698-705.

85. Nelson KE, Fleischmann RD, DeBoy RT y cols. Complete genome sequence of the oral pathogenic bacterium Porphyromonas gingivalis strain W83. Journal of Bacteriology. 2003; 185: 5591-601.

86. Chen T, Hosogi Y, Nishikawa K y cols. Comparative whole-genome analysis of virulent and avirulent strains of Porphyromonas gingivalis. Journal of Bacteriology. 2004; 186: 5473-9. 